\title{
Fractional quantum Hall states in two-dimensional electron systems with anisotropic interactions
}

\author{
Hao Wang, ${ }^{1}$ Rajesh Narayanan, ${ }^{1,2,3}$ Xin Wan, ${ }^{4}$ and Fuchun Zhang ${ }^{1,4}$ \\ ${ }^{1}$ Department of Physics, The University of Hong Kong, Hong Kong SAR, China \\ ${ }^{2}$ Department of Physics, Indian Institute of Technology Madras, Chennai 600036, India \\ ${ }^{3}$ Department of Physics, Hong Kong University of Science and Technology, Hong Kong SAR, China \\ ${ }^{4}$ Zhejiang Institute of Modern Physics, Zhejiang University, Hangzhou 310027, China \\ (Received 26 March 2012; revised manuscript received 18 June 2012; published 16 July 2012)
}

\begin{abstract}
We study the anisotropic effect of the Coulomb interaction on a 1/3-filling fractional quantum Hall system by using an exact diagonalization method on small systems in torus geometry. For weak anisotropy the system remains to be an incompressible quantum liquid, although anisotropy manifests itself in density correlation functions and excitation spectra. When the strength of anisotropy increases, we find the system develops a Hallsmectic-like phase with a one-dimensional charge density wave order and is unstable towards the one-dimensional crystal in the strong anisotropy limit. In all three phases of the Laughlin liquid, Hall-smectic-like, and crystal phases the ground state of the anisotropic Coulomb system can be well described by a family of model wave functions generated by an anisotropic projection Hamiltonian. We discuss the relevance of the results to the geometrical description of fractional quantum Hall states proposed by Haldane [Phys. Rev. Lett. 107, 116801 (2011)].
\end{abstract}

DOI: 10.1103/PhysRevB.86.035122

PACS number(s): 73.43.Cd, 73.43.Nq, 71.10.Pm

\section{INTRODUCTION}

The fractional quantum Hall $(\mathrm{FQH})$ effect at an odd denominator filling of $v$ has been understood as a property of an incompressible quantum liquid in an interacting twodimensional (2D) electron system. Laughlin's trial wave function ${ }^{1}$ is the first successful theory to describe this manybody effect, where the interacting system is implicitly assumed to be isotropic. Since then most theoretical works on the FQH system have followed this simple assumption, and the FQH states are considered to be isotropic with rotational symmetry.

However, the real FQH systems may be anisotropic. One natural source for this is the anisotropic dielectric tensor, which in turn leads to an anisotropic Coulomb interaction. Other mechanisms for various anisotropic $\mathrm{FQH}$ systems have also been discussed theoretically ${ }^{2-6}$ and experimentally. ${ }^{7,8}$ For example, an anisotropic $\mathrm{FQH}$ state in a $v=7 / 3$ system has been observed in experiment. ${ }^{8}$ In these anisotropic FQH systems, rotational symmetry of the consequent ground state is expected to be broken. In the extreme anisotropic interaction limit, where the Coulomb interaction can be effectively treated to be one-dimensional (1D), the ground state of the system will be a quasi-1D crystal. ${ }^{9}$ Thus, the properties of the FQH state in an anisotropic interaction may not always be associated with the isotropic incompressible liquid. A comprehensive investigation on the effect of the interaction anisotropy is called for.

Motivated by the anisotropic transport properties experimentally reported at the partially filled higher Landau level (LL), trial wave functions ${ }^{10-13}$ have been proposed to describe the anisotropic $\mathrm{FQH}$ states. These variational wave functions modify the original isotropic Laughlin wave function by splitting the multiple-order zeros in the wave function. Very recently, Haldane ${ }^{2}$ has constructed a family of the Laughlin states, which are the exact ground states of the corresponding projected Hamiltonians and can be parameterized according to the interaction anisotropy. This variational state can be compared numerically with the anisotropic FQH state. An effort to map the underlying wave function of this variational state has been reported. ${ }^{5}$ In this paper we study FQH states in 2D electron systems with anisotropic Coulomb interaction and discuss the relevance of our results with the geometric description of the FQH states. ${ }^{2}$

The paper is organized as follows. In Sec. II we introduce our model with anisotropic Coulomb interaction and set up the Hamiltonian on a torus geometry. In Sec. III we discuss the properties of $v=1 / 3 \mathrm{FQH}$ states at different regimes of the interaction anisotropy using energy spectra, charge density, and correlation functions. We also compare the anisotropic FQH state with variational Laughlin states using wave-function overlap. Section IV summarizes the paper.

\section{MODEL AND NUMERICAL SETUP}

We study a 2D electron system under a perpendicular magnetic field $\mathbf{B}=B \hat{z}$. The electron-electron Coulomb interaction with an in-plane biaxial dielectric tensor has the form

$$
V_{c}(\mathbf{r})=\frac{e^{2}}{4 \pi \varepsilon \sqrt{A_{c} x^{2}+y^{2} / A_{c}}},
$$

where $A_{c}$ is the interaction anisotropy parameter, and the directions of $\hat{x}$ and $\hat{y}$ are along the two principal axes of the dielectric tensor. The effective mass tensor is considered isotropic so that noninteracting electrons move in the circular cyclotron orbitals. However, equipotential lines of the Coulomb interaction are generally elliptical with $A_{c} \neq 1$. In the following discussion, we choose $A_{c} \geqslant 1$ such that $\hat{x}$ is the hard axis. For $A_{c}<1$, one simply swaps the easy and hard axes. At $A_{c} \gg 1$, the Coulomb interaction is effectively a 1D repulsion along the hard axis.

In our numerical calculations, we use Landau gauge $(0, B x)$ for the magnetic vector potential. Periodic boundary conditions for the magnetic translational operators are imposed with a quantized flux $N_{\phi}$ through the rectangular unit cell $\mathbf{L}_{\mathbf{x}} \times \mathbf{L}_{\mathbf{y}}$. The magnetic length $\ell$ is taken as the unit length 
and the energy is in units of $e^{2} / 4 \pi \varepsilon \ell$. To reduce the size of the Hilbert space, we carry out our calculation at every pseudomomentum $\mathbf{K}=\left(K_{x}, K_{y}\right),{ }^{14}$ where $K_{x}\left(K_{y}\right)$ is in units of $2 \pi / L_{x}\left(2 \pi / L_{y}\right)$. The magnetic field is assumed to be strong enough so that the spin degeneracy of the Landau levels is lifted. ${ }^{14,15}$ One can thus project the system Hamiltonian into the valence Landau level. ${ }^{14}$ For the lowest Landau level, the projected Hamiltonian has the form

$$
H_{c}=\frac{1}{N_{\phi}} \sum_{\mathbf{q}} V(\mathbf{q}) e^{-q^{2} / 2} \sum_{i<j} e^{i \mathbf{q} \cdot\left(\mathbf{R}_{i}-\mathbf{R}_{j}\right)},
$$

where the momentum $\mathbf{q}=\left(q_{x}, q_{y}\right)$ takes discrete values suitable for the lattice of the unit cell, and $\mathbf{R}_{i}$ is the guiding center coordinate of the $i$ th electron. $V(\mathbf{q})=1 / \sqrt{q_{x}^{2} / A_{c}+A_{c} q_{y}^{2}}$ is the Fourier transform of the Coulomb interaction. From the geometrical point of view, we generalize $q^{2}=q_{x}^{2}+q_{y}^{2}$ to $q_{g}^{2}=g^{a b} q_{a} q_{b}$, where

$$
g=\left(\begin{array}{cc}
1 / A_{c} & 0 \\
0 & A_{c}
\end{array}\right)
$$

is the inverse metric for the Coulomb interaction.

\section{NUMERICAL RESULTS AND DISCUSSION}

For a $v=1 / 3 \mathrm{FQH}$ system, we first study the low-lying energy spectra using an exact diagonalization method. Here and in following subsections, the default size of the $v=1 / 3$ system is $N_{e}=10$, and the default shape of the unit cell is square unless otherwise specified. We find qualitatively similar results in systems with other sizes and/or different shapes of the unit cell.

Figure 1 plots the excitation energy gap as a function of the Coulomb interaction anisotropy. In the range up to $A_{c}=100$ the curve is nonmonotonic and develops several distinct regimes. For small interaction anisotropy up to

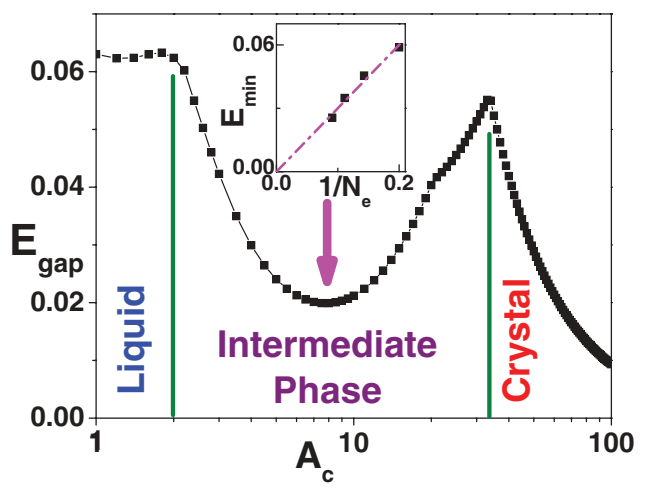

FIG. 1. (Color online) Excitation energy gap versus interaction anisotropy for an $N_{e}=10$ and $v=1 / 3 \mathrm{FQH}$ system with a square unit cell. The horizontal axis is plotted in the log scale to show the transition at low anisotropy. The system is in a Laughlin-liquid-like state for $A_{c}<2.0$ (Sec. III A), and becomes a quasi-1D crystal for $A_{c}>33.0$ (Sec. III C), whose excitation gap scales as $1 / \sqrt{A_{c}}$. The intermediate regime is related to a Hall-smectic-like phase, which is unstable towards the quasi-1D crystal and will be discussed in Sec. III B. The inset shows a linear size-scaling for the minimum gap in the intermediate regime.
$A_{c}=2.0$, the energy gap remains nearly constant, indicating the incompressible liquid phase in the isotropic case (i.e., $A_{c}=1$ ) is robust against weak interaction anisotropy. When the interaction anisotropy further increases, the energy gap decreases to a minimum at around $A_{c}=8.0$. For larger $A_{c}$ the energy gap increases with the interaction anisotropy to a maximum at around $A_{c}=33.0$. The finite-size scaling shown in the inset reveals that the minimum gap can close in the thermodynamic limit, suggesting there might exist a switch between different order parameters ruling the system. Beyond $A_{c}=33.0$ the energy gap decreases roughly as $1 / \sqrt{A_{c}}$, indicating the regime of the quasi-1D repulsion limit. We have studied other system sizes and found that these boundaries are size dependent. But, in general, the ground state of the system maintains a threefold degeneracy. This adiabatic transition with complex regimes typically occurs between distinct phases with a competition in the intermediate region. In the following subsections, we will focus on these different regimes in $A_{c}$ and reveal an interesting competition between liquid and crystal phases.

\section{A. Anisotropic Laughlin liquid at small interaction anisotropy}

The energy gap plot suggests that the ground state at small interaction anisotropy is an incompressible liquid similar to the isotropic Laughlin state. The anisotropy in interaction, however, is expected to be imprinted, e.g., in the static structure factor of the resulting incompressible liquid. The projected static structure factor is defined as ${ }^{14}$

$$
S_{0}(\mathbf{q})=\frac{1}{N_{e}}\left\langle 0\left|\sum_{i \neq j} e^{i \mathbf{q} \cdot\left(\mathbf{r}_{i}-\mathbf{r}_{j}\right)}\right| 0\right\rangle,
$$

where $|0\rangle$ is the calculated ground state and $\mathbf{r}_{i}$ is the coordinate of the $i$ th particle.

In Fig. 2(a), we draw the three-dimensional (3D) and contour plots of the structure factor for a calculated $\mathrm{FQH}$ state at $A_{c}=1.8$. It exhibits a craterlike feature, which is similar to that of the isotropic liquid. However, the overall shape of the crater is deformed, stretching along the hard axis direction. Therefore, the elliptical symmetry replaces the circular symmetry in the isotropic liquid case.

This anisotropic signature is more prominent in the $2 \mathrm{D}$ cuts along the two principal axes as shown in Fig. 2(b). We note that the structure factor behaves asymptotically as $\sim q^{4}$ in the long wavelength limit. This agrees with the single mode approximation ${ }^{16}$ (SMA) for an incompressible liquid. However, the prefactor of the quartic term is orientation dependent, revealing the anisotropic nature of the structure factor. According to Ref. 6, the ratio of prefactors at the $q_{x}$ and $q_{y}$ axes is equal to $\left(1 / A_{L}^{*}\right)^{4}$, where the parameter $A_{L}^{*}$ defines an intrinsic metric, describing how the correlated quasiparticles bind to each other in the anisotropic environment. The fitting lines in the plot have revealed $A_{L}^{*} \sim 1.45$ for interaction anisotropy $A_{c}=1.8$. The peaks in the orientation-dependent plots represent the crater ridge in Fig. 2(a).

According to the SMA, the maximum in the structure factor corresponds to a minimum gap in the excitation spectrum, or the roton minimum, which corresponds to the excitonic binding of the neutral quasiparticle-quasihole pairs. ${ }^{16}$ This is evident in Fig. 2(c), where we plot the orientation-dependent 

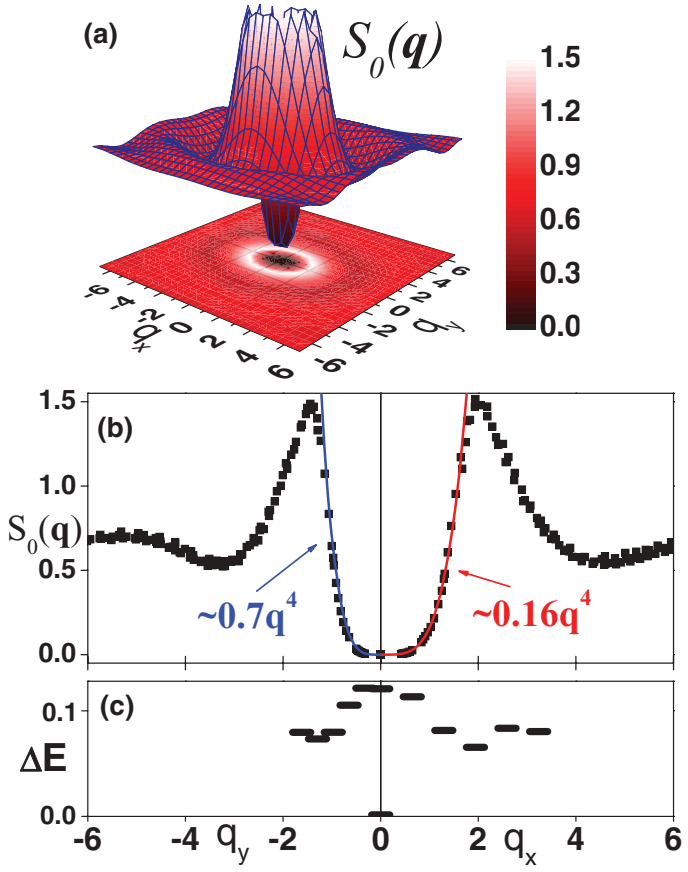

FIG. 2. (Color online) (a) 3D and contour plots of the structure factor, (b) the structure factor along the $q_{x}$ and $q_{y}$ axes, and (c) the excitation spectrum along the $q_{x}$ and $q_{y}$ axes for the $N_{e}=10$ system with Coulomb interaction anisotropy $A_{c}=1.8$. To overcome the discrete momentum limitation, we use unit cells with different aspect ratios $R_{a}=L_{x} / L_{y}$ in (b) to obtain more data points and a unit cell with $R_{a}=0.5$ in (c).

low-energy excitation spectra in the momentum space. The location of the roton minimum is sensitive to the direction, but the gap value is less sensitive. The ratio of the roton-minimum locations along the $q_{x}$ and $q_{y}$ axes is found close to $A_{L}^{*}$ as expected, and these two locations match the peak locations of the structure factor in Fig. 2(b).

The isotropic Laughlin wave function, with order-3 zeros at the locations of other particles, triumphed in the explanation of the isotropic incompressible liquids of the $v=1 / 3$ FQH system. Corresponding to the deformed electron-hole correlation from the anisotropic interaction, order-3 zeros in the wave function are expected to split. Several works have suggested that the relative coordinate part of the anisotropic wave function ${ }^{5,10-13}$ has the form

$$
w\left(z_{i}\right) \sim \prod_{i<j}\left(z_{i}-z_{j}\right)\left[\left(z_{i}-z_{j}\right)^{2}+z_{0}^{2}\right],
$$

where $z_{i}$ is the complex coordinate of the $i$ th particle and $z_{0}$ is a complex constant related to the splitting of the zeros due to anisotropy. This zero-splitting effect in the wave function can be detected using the pair correlation function defined as ${ }^{17}$

$$
g(\mathbf{r})=\frac{L_{x} L_{y}}{N_{e}\left(N_{e}-1\right)}\left\langle 0\left|\sum_{i \neq j} \delta\left(\mathbf{r}-\left(\mathbf{r}_{i}-\mathbf{r}_{j}\right)\right)\right| 0\right\rangle .
$$

In Fig. 3(a), we plot the pair correlation functions along the $\hat{x}$ and $\hat{y}$ directions for the $v=1 / 3 \mathrm{FQH}$ state with the interaction anisotropy $A_{c}=1.8$. The two curves are distinguishable from their isotropic counterparts. We point out that the curves behave asymptotically as $\alpha r^{2}$ in the limit of $r \rightarrow 0$, with the

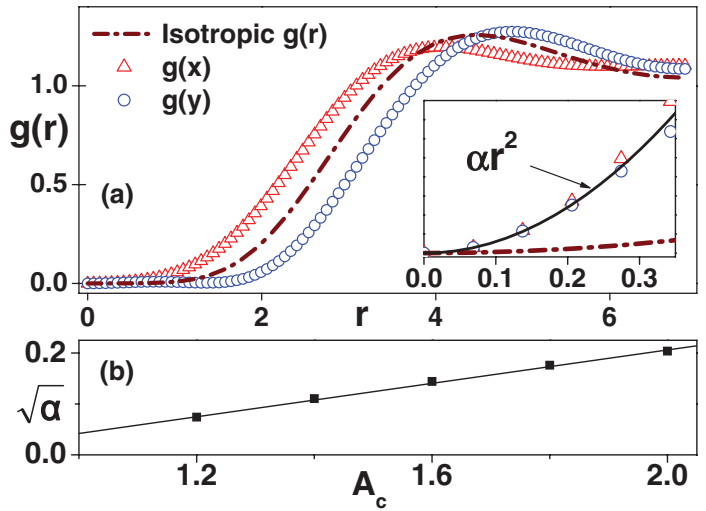

FIG. 3. (Color online) (a) Pair correlation function along the $\hat{x}$ and $\hat{y}$ axes for the $N_{e}=10$ system at interaction anisotropy $A_{c}=1.8$. The dotted-dashed line represents the correlation function at $A_{c}=1$ for comparison. The inset reveals the $\alpha r^{2}$ behavior at small $r$ for the anisotropic case. (b) A linear fit for the prefactor $\sqrt{\alpha}$ at small anisotropy.

prefactor $\alpha \propto\left|z_{0}\right|^{4}$. This is entirely different from the isotropic Laughlin wave function, which exhibits an $r^{6}$ asymptotic behavior in its pair correlation function. The nonmonotonic behavior in $g(y)$ at the small $r$ region, which manifests itself more clearly at a larger $A_{c}$, is also consistent with the zero-splitting scenarios. ${ }^{5,13}$ We point out that for a suitable deformed model wave function, there is also an additional contribution to the Gaussian Landau level form factor, 5,18 which can be observable in the disk geometry with a boundary.

In Fig. 3(b), we plot the square root of the prefactor $\alpha$ at several small anisotropy. The linear fit of $\sqrt{\alpha}$ to $A_{c}$ is expected as $\left(A_{c}-1\right)$ [or $\left.\left(\sqrt{A_{c}}-1\right)\right]$, which characterizes the perturbation away from the isotropic point. However, the resulting nonzero intercept at $A_{c}=1$ suggests that we may have overestimated the prefactor, possibly due to the higher order contributions at small $A_{c}$.

\section{B. Hall-Smectic-like phase in the intermediate interaction anisotropy regime}

The explicit construction ${ }^{5}$ of the model wave function by unimodular transformation on the disk geometry suggests that the geometrical description of the quantum Hall system accepts the following deformation of the isotropic Laughlin state (i.e., $\gamma=0)$ :

$$
\Psi=\prod_{i<j} z_{i j}\left[z_{i j}^{2}+\frac{12 \gamma^{*}}{1-|\gamma|^{2}}\right] e^{-\sum_{i} \gamma z_{i}^{2} / 4} e^{-\sum_{i}\left|z_{i}\right|^{2} / 4},
$$

where $z_{i j}=z_{i}-z_{j}$ and $\gamma$ characterizes the amount of mixing between the guiding center creation and annihilation operators in the unimodular transformation. Note that the model wave function is expected to be valid for small $\gamma$. In the present parametrization $\gamma=\sqrt{A_{L}^{*}}-1$ is real. We can postulate the breakdown criterion for the anisotropic Laughlin liquid to be

$$
\pi \frac{12 \gamma^{*}}{1-|\gamma|^{2}}=\frac{2 \pi}{v},
$$

i.e., the area occupied by a set of three splitting zeros is the average area per particle. This suggests that at the breakdown 

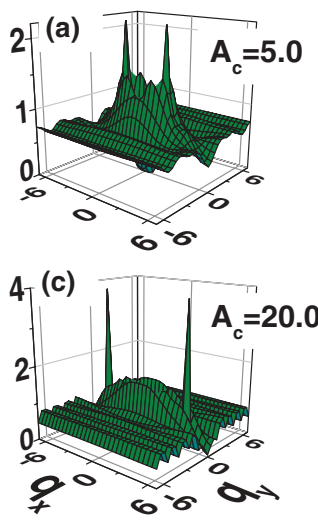
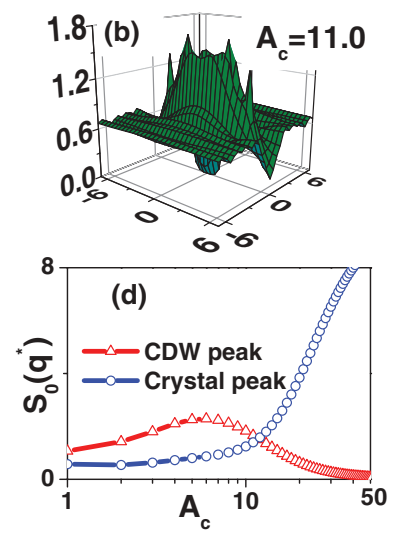

FIG. 4. (Color online) (a)-(c) Structure factors for the ground states of the $N_{e}=10$ system at $A_{c}=5.0,11.0$, and 20.0, respectively. Note that the locations of the twin peaks for $A_{c}=5.0$ (CDW-like peaks) differ from those for $A_{c}=20.0$ (crystal peaks). Both types of peaks coexist at $A_{c}=11.0$. (d) Peak values of the CDW-like and crystal peaks are plotted as a function of the interaction anisotropy in a range of $1<A_{c}<50$.

$A_{L}^{*} \approx 2$ (i.e., $A_{c} \approx 3$ according to the estimation in the Sec. IIID), consistent with the onset of the rapid decrease of the excitation gap.

In other words, the anisotropic Laughlin liquid is stable when the long-distance (i.e., at average particle spacing) behavior of the Jastrow factor is still as $z_{i j}^{1 / \nu}$. The collective excitation of the liquid is the neutral magnetoroton excitations, which becomes anisotropic. When the liquid phase breaks down, it cannot sustain further anisotropy by the spatial deformation in the roton spectrum.

One possible outcome of the system after this breakdown is that the mode at the roton minimum goes softer, developing some charge-density-wave (CDW) order. Due to the orientation effect of the anisotropy, this CDW is expected to be unidirectional (stripelike), and the characterizing sharp peaks in the structure factor are along the stretching direction. This is clearly visible in the structure factor at $A_{c}=5.0$ in Fig. 4(a). The background in the structure factor resembles that of an anisotropic Laughlin liquid, but its peak value is significantly smaller than the two sharp peaks along the $q_{x}$ axis. The CDW twin peaks correspond to a period in real space, which can be roughly anticipated as the splitting of zeros $\left|z_{0}\right|=\sqrt{6}$ at the critical $A_{L}^{*}=2$. The peak value (subtracting background) in the structure factor suffices as the order parameter. The plot in Fig. 4(d) shows that this CDW-like order parameter rules the system in the regime $2.0<A_{c}<8.0$.

We term this phase, which breaks one-dimensional translational symmetry, as a Hall-smectic-like phase since we speculate that it is related to the Hall smectic discussed earlier in the context of liquid crystal phases in the $\mathrm{FQH}$ system. ${ }^{10-12,19-21}$ The rise of the smectic phase softens the magnetoroton mode and appears to be responsible for the reduction of the excitation gap for $2.0<A_{c}<8.0$ as shown in Fig. 1. As discussed in Ref. 11 the transition from the Laughlin liquid to the Hall smectic can be second order and its critical behavior is in the $X Y$ universality class.

Beyond $A_{c}=8.0$, the reverse trend in the excitation gap as a function of $A_{c}$ indicates that the system is under the influence of a distinct mechanism. This is evidently shown in the structure factor plot of Fig. 4(b) at $A_{c}=11.0$. Two additional peaks along the $q_{x}$ axis are clearly visible with the different wave vectors from the CDW-like twin peaks. These additional twin peaks are corresponding to the unidirectional crystal order in the quasi-1D repulsion limit that we will discuss in Sec. IIIC. The peak value of them is plotted as the crystal order parameter in Fig. 4(d). There we can see that the crystal order parameter is continuously increasing with the interaction anisotropy. The crossover for the competition with the CDW-like order occurs around $A_{c}=13.0$. For larger anisotropy the crystal order dominates as illustrated in Fig. 4(c) at $A_{c}=20.0$, where only the crystal peaks remain. Thus, the Hall-smectic-like phase is found unstable towards a 1D crystal.

\section{Quasi-1D crystal in the large anisotropy limit}

The crystal phase at the large anisotropy limit can be probed using the charge distribution. In Fig. 5(a), we plot the average LL orbital occupations and the charge density along the hard axis at $A_{c}=40.0$. The charge density appears smoother as an integral from local Gaussian wave packets over orbital occupations, and a $2 \%$ fluctuation above the background can be observed in the exaggerated plot. Both the charge occupation and density fluctuate along the hard axis with the crystalline period $\lambda^{*}=L_{x} / N_{e}$. The maximum charge occupation is close to unity as expected in the ultimate 1D crystal limit. The $2 \mathrm{D}$ distribution of the charge density reveals that the system is a unidirectional crystal with each electron spreading into a stripe perpendicular to the hard axis. For basis states in the torus geometry, the guiding-center coordinate along the hard axis is coupled with the momentum along the $q_{y}$ axis. ${ }^{17}$ Thus, the calculated ground states are expected to carry a period of $2 \pi / \lambda_{y}$ in the momentum space along the $q_{y}$
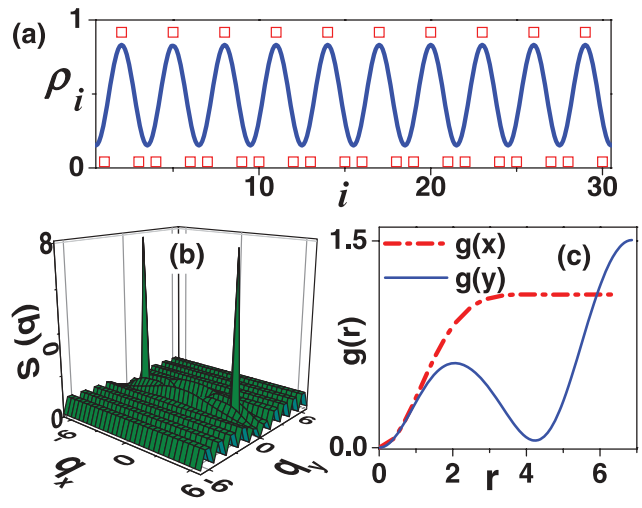

FIG. 5. (Color online) For ground states of the $N_{e}=10$ system at $A_{c}=40.0$. (a) Average occupations (scattered squares) of the Landau-level orbital at guiding centers and the charge density (solid line) are plotted along the hard axis. A period of $\lambda^{*}=L_{x} / N_{e}$ is clearly visible. The density values have been shifted and exaggerated to emphasize a $\sim 2 \%$ fluctuation over the background. (b) Structure factor with sharp twin peaks in the $\hat{q}_{x}$ direction and periodic oscillation in the $\hat{q}_{y}$ direction. (c) Pair correlation function is plotted along two principal axes. The function $g(y)$ shows an oscillation with the period $L_{y} / 3$. 
axis, where $\lambda_{y}=L_{y} / 3$. The structure factor plot in Fig. 5(b) demonstrates this characteristic order along $q_{y}$ axis. As its real space counterpart, the pair correlation plot in Fig. 5(c) shows oscillation in the $\hat{y}$ direction with a period $\lambda_{y}$.

The above results support that at filling $v=1 / 3$ the ground state of the system is a crystal in the large interaction anisotropy limit and the system undergoes some transition from an incompressible liquid to a solid as anisotropy increases. A similar story has been discussed in an isotropic FQH system with extreme geometry, such as in a thin torus or a cylinder limit, ${ }^{2-24}$ and in a recent work ${ }^{25}$ on the graphene ribbon with flat bands. They can be explained under the same principle in Ref. 24 by a sorting Hamiltonian. When the interaction anisotropy increases, the repulsion-related diagonal terms dominate, which has the similar effect as geometry on the isotropic $\mathrm{FQH}$ and as the local orbital expansion on the flat-band graphene ribbon. The low-energy physics is governed by the strong repulsion so that the system tends to form crystal. At small anisotropy, the hopping-related off-diagonal terms are comparable and screen the repulsion, resulting in the liquid phase.

\section{Generalized variational Laughlin state}

In the discussion above, we have seen that the isotropic Laughlin wave function is insufficient to fully capture the features of an anisotropic FQH system. For such a system at the lowest LL filling $v=1 / q$, Haldane has suggested to use a family of Laughlin states, ${ }^{2}$ which is generally defined as the densest zero-energy eigenstate of a projected two-body anisotropic Hamiltonian:

$$
H_{v}\left(A_{L}\right)=\sum_{m<q} P_{m}\left(A_{L}\right) .
$$

For the fermion system with an odd denominator $q, m$ are limited to be odd. This Hamiltonian is a truncated summation over anisotropic pair interactions:

$$
P_{m}\left(A_{L}\right)=\frac{1}{N_{\phi}} \sum_{\mathbf{q}} L_{m}\left(Q^{2}\right) e^{-Q^{2} / 2} \sum_{i<j} e^{i \mathbf{q} \cdot\left(\mathbf{R}_{i}-\mathbf{R}_{j}\right)}
$$

for two particles with the relative angular momentum of $m \hbar$ in the guiding-center coordinates. In the above expression, $L_{m}(x)$ are $m$ th Laguerre polynomials and $Q\left(A_{L}\right)=$ $\sqrt{q_{x}^{2} / A_{L}+A_{L} q_{y}^{2}}$, which, like in Eq. (3), defines a wavefunction metric parameterized by $A_{L}$. The parameterized Laughlin states $\Psi\left(A_{L}\right)$ satisfy

$$
P_{m}\left(A_{L}\right)\left|\Psi\left(A_{L}\right)\right\rangle=0, m<q .
$$

The isotropic Laughlin wave function corresponds to the Laughlin state with $A_{L}=1$. With this family of parameterized states, we are able to variationally approximate the ground state of a FQH system with anisotropic interaction. According to Haldane's proposal, if the mass or orbital metric (in our case, an identity matrix for isotropic mass) is different from the interaction metric (parameterized by $A_{c}$ ), the resulting variational state $\Psi\left(A_{L}^{*}\right)$ should be described by a metric interpolating the mass metric and the interaction metric, i.e., $1<A_{L}^{*}<A_{c}$. This intrinsic metric describes how correlated

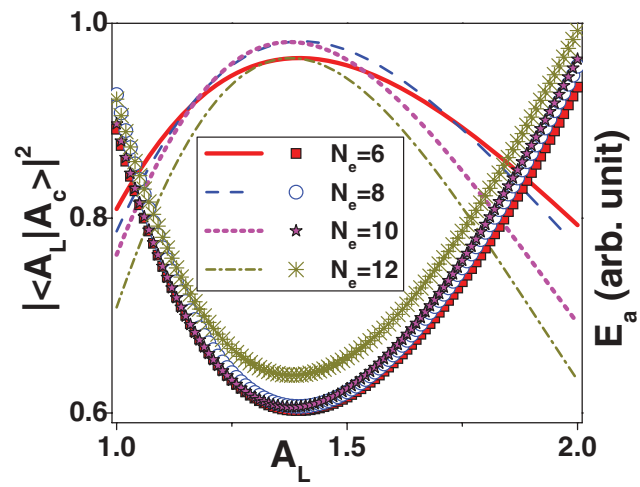

FIG. 6. (Color online) Wave-function square overlap (lines) and expected value of Coulomb energy (symbols) as a function of the variational parameter $A_{L}$ for the $v=1 / 3 \mathrm{FQH}$ system at interaction anisotropy $A_{c}=1.8$. The energy values have been shifted and enlarged to emphasize that the location of the minimum coincides with that of the largest overlap. The comparison of different sizes of $N_{e}=6,8,10$, and 12 shows a weak size dependence only.

quasiparticles effectively feel each other in such an anisotropic FQH system.

In Fig. 6, we study the anisotropic $v=1 / 3 \mathrm{FQH}$ system with the Coulomb anisotropy $A_{c}=1.8$. The optimal Laughlin state $\Psi\left(A_{L}^{*}\right)$ is obtained by tracing either the maximum of the wave-function overlap or the minimum of the expected Coulomb energy

$$
E_{a}\left(A_{L}\right)=\left\langle\Psi\left(A_{L}\right)\left|H_{c}\left(A_{c}\right)\right| \Psi\left(A_{L}\right)\right\rangle .
$$

The optimal parameter is found at $A_{L}^{*} \sim 1.43$, which is weakly size dependent. This parameter is indeed an intermediate value between unity and the Coulomb anisotropy as expected. ${ }^{2}$ It also agrees with the intrinsic metrics through the analysis of the anisotropic structure factor in the Sec. III A. The overlaps between the optimal Laughlin state, and the exact ground states are larger than $90 \%$ for various system sizes, which supports the validity of the variational state. We also note that the expected Coulomb energy quadratically approaches its minimum, which suggests a linear approximation of the anisotropic Laughlin state with $A_{L}$ in the liquid phase regime.

To gain a further understanding for the validity of this variational approach, we approximately expand the Coulomb interaction in the anisotropic pair interactions as

$$
H_{c}\left(A_{c}\right) \approx \sum_{m} V_{m}\left(A_{c}, A_{L}\right) P_{m}\left(A_{L}\right),
$$

where the average expansion coefficients $V_{m}$ define the effective anisotropic pseudopotentials in a form of

$$
V_{m}=\int_{0}^{2 \pi} d \theta \int_{0}^{\infty} d x \frac{L_{m}\left(x^{2}\right) e^{-F(\theta, x) / 2}}{2 \pi \sqrt{G(\theta)}}
$$

with

$$
G(\theta)=\left(A_{c} / A_{L}\right) \cos ^{2} \theta+\left(A_{L} / A_{c}\right) \sin ^{2} \theta
$$

and

$$
F(\theta, x)=x^{2}\left(1+A_{L} \cos ^{2} \theta+\sin ^{2} \theta / A_{L}\right) .
$$

An example of $V_{m}$ is plotted in Fig. 7(a) with $A_{c}=1.8$ and $A_{L}=1.47$. These pseudopotential parameters are found 

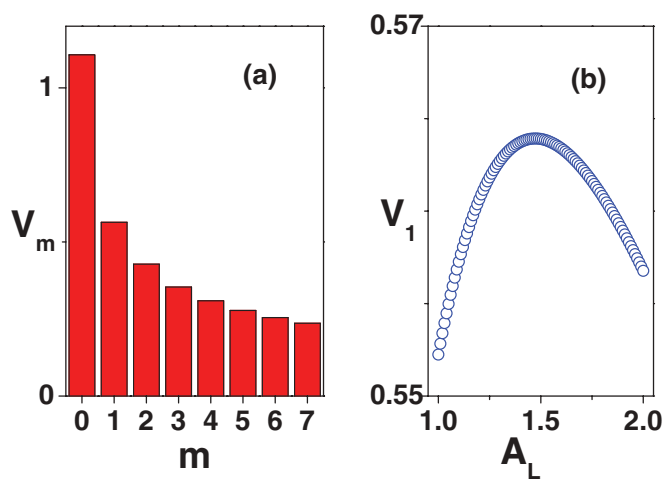

FIG. 7. (Color online) For a Coulomb system with interaction anisotropy $A_{c}=1.8$. (a) Effective pseudopotentials $V_{m}$ with a given parameter $A_{L}=1.47$ is plotted against $m$. (b) The pseudopotential $V_{1}$ is plotted as a function of $A_{L}$. The maximum value occurs around $A_{L}=1.47$.

positive and monotonously decrease with $m$, consistent with the long range behavior of the Coulomb repulsion.

Given the Coulomb interaction anisotropy $A_{c}$, in principle we can have a family of pseudopotential sets parameterized by $\left\{A_{L}\right\}$, which are associated with different values. The set of $V_{m}$ with the maximum pseudopotential values up to the $m$ th order is most promising for the truncation-based variational approach to work. Thus, for the $v=1 / 3$ system, we could use the maximum of $V_{1}$ as a criterion to examine the optimal parameter of the variational Laughlin state, $A_{L}^{*}$. This sizeindependent condition

$$
V_{1}\left(A_{L}^{*}\right) \geqslant V_{1}\left(A_{L}\right)
$$

serves as a semianalytic estimation to the intrinsic geometry parameter $A_{L}^{*}$ of the $v=1 / 3$ anisotropy system. As shown in Fig. 7(b), the estimated optimal parameter is around $A_{L}^{*}=$ 1.47 , matching the value found previously through the finitesize calculation.

In the 2D contour plot of Fig. 8, we show a comprehensive map of the wave-function overlap as a function of both parameters $A_{c}$ and $A_{L}$. The isotropic Laughlin wave function

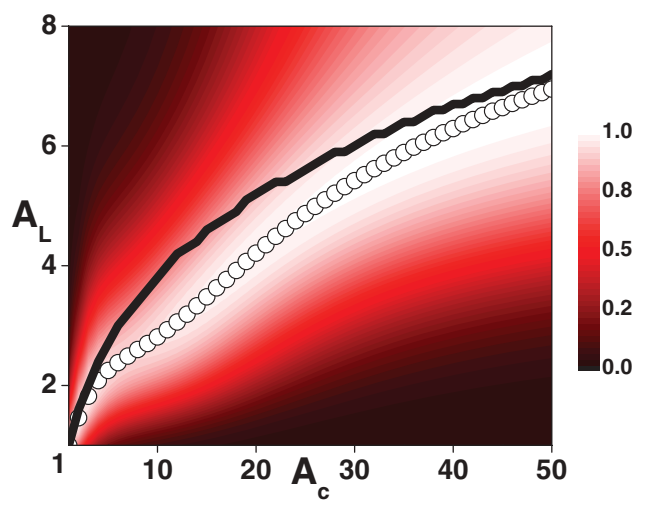

FIG. 8. (Color online) Contour plot of wave-function square overlap between the Coulomb ground state (parameterized by $A_{c}$ ) and the generalized Laughlin state (parameterized by $A_{L}$ ) for the $N_{e}=10$ system. Each dot represents the maximum of the wave-function overlap for a given $A_{c}$. The solid curve stands for the estimated fit $A_{L}^{*}$ as a function of $A_{c}$ using the maximum $V_{1}$ criterion. has a continuously decreasing overlap with the calculated $\mathrm{FQH}$ state when the Coulomb anisotropy increases, indicating again its insufficiency in describing the anisotropic FQH system. Instead, we note that at the optimal parameters, the local maximum of the wave-function overlap is larger than $90 \%$ in the full range of the Coulomb anisotropy parameter (even when the system is not in the liquid phase). This justifies that the family of variational Laughlin states, which have larger wave-function overlaps, could serve a better description of the anisotropically interacting FQH system. We also notice that in the vicinity of $A_{c}=1$, the optimal parameter of the Laughlin state is linearly related to the interaction anisotropy. As a comparison, we plot the estimated optimal parameters $A_{L}^{*}$ from Eq. (17) as a function of $A_{c}$. We find they agree well with the finite-size results in the liquid and crystal phases. But in the intermediate regime the results show some deviation. This suggests a more careful handling beyond the simple criterion of Eq. (17) is needed for the intermediate region.

\section{SUMMARY}

In conclusion, we have studied the effect of anisotropic Coulomb interaction on the ground state of the 1/3 filling fractional quantum Hall system. We find that at weak anisotropy the Laughlin state remains to be a valid description, although the structure factor and pair correlation function exhibit anisotropy. Our calculations support the recent proposal of Haldane on the geometric description of the fractional quantum Hall state. ${ }^{2}$ In particular, the order- 3 zeros in the wave function split into three distinct zeros with a splitting distance related to the anisotropy. ${ }^{5}$ We have compared the ground state wave function of the anisotropic Coulomb interaction with a family of single-parameter variational Laughlin states. The latter are obtained by deforming the projection (i.e., $V_{1}$ only) Hamiltonian for the isotropic Laughlin state. We have determined the variational parameter by minimizing the variational ground state energy or by maximizing the wave-function overlap. In addition, we also propose an effective analysis to estimate the optimal variational parameter. The liquid phase breaks down when anisotropy increases and a Hall-smectic-like order emerges. Finally, at strong interaction anisotropy, which is more of theoretical interest, the ground state exhibits a compressible one-dimensional crystal phase. Interestingly, the ground state obtained by solving the deformed projection Hamiltonian remains to be a good description (overlap greater than $90 \%$ ) throughout the liquid to solid transition.

The anisotropy in the Coulomb interaction provides a new route in probing the intrinsic metric of the fractional quantum Hall state in its geometrical description, as pointed out by Haldane. ${ }^{2}$ In the present study we have revealed that the intrinsic metric or the wave-function anisotropy are indeed different from the Coulomb metric or the dielectric tensor anisotropy, although they appear to be linearly proportional to each other in the vicinity of the isotropic Laughlin state. The linearity we have found supports the proposal to use a single-parameter to construct unimodularly deformed wave functions to describe the effect of interaction anisotropy in the disk geometry. ${ }^{5}$ In fact, the family of deformed wave functions contain the same anisotropic Jastrow factor in their relative coordinate part, ${ }^{10}$ which explicitly splits the order- 3 
zeros in the Laughlin liquid. Therefore, the emergence of the Hall smectic phase, as analyzed in the effective field theories, ${ }^{11,12}$ at larger anisotropy is also a support of the geometrical description of FQH states.

Near the completion of this work, we note a very recent work $^{26}$ which discusses the anisotropic FQH system with the anisotropic band mass. There, the persistent energy gap and anisotropic roton-minimum excitation at small anisotropy parameters are reported, consistent with our results in the liquid phase.

\section{ACKNOWLEDGMENTS}

We thank Weiqiang Chen for discussions. X.W. thanks Ruizhi Qiu and Su Yi for a related collaboration on an anisotropic dipolar interaction in ultracold fermion systems. This work was supported by an RGC grant in Hong Kong, the National Basic Research Program of China (973 Program) Grant No. 2012CB927404, the National Natural Science Foundation of China (NSFC) Grant No. 11174246, and the DST India Project SR/S2/HEP-012/2009.
${ }^{1}$ R. B. Laughlin, Phys. Rev. Lett. 50, 1395 (1983).

${ }^{2}$ F. D. M. Haldane, Phys. Rev. Lett. 107, 116801 (2011).

${ }^{3}$ R. Z. Qiu, S. P. Kou, Z. X. Hu, X. Wan, and S. Yi, Phys. Rev. A 83, 063633 (2011).

${ }^{4}$ M. Mulligan, C. Nayak, and S. Kachru, Phys. Rev. B 82, 085102 (2010); 84, 195124 (2011).

${ }^{5}$ R. Z. Qiu, F. D. M. Haldane, X. Wan, K. Yang, and S. Yi, Phys. Rev. B 85, 115308 (2012).

${ }^{6}$ F. D. M. Haldane, arXiv:1112.0990.

${ }^{7}$ K. K. Ni, S. Ospelkaus, M. H. G. de Miranda, A. Pe'er, B. Neyenhuis, J. J. Zirbel, S. Kotochigova, P. S. Julienne, D. S. Jin, and J. Ye, Science 322, 231 (2008).

${ }^{8}$ J. Xia, J. P. Eisenstein, L. N. Pfeiffer, and K. W. West, Nat. Phys. 7, 845 (2011).

${ }^{9}$ M. Aizenman, S. Jansen, and P. Jung, Ann. Henri Poincaré 11, 1453 (2010).

${ }^{10}$ K. Musaelian and R. Joynt, J. Phys.: Condens. Matter 8, L105 (1996).

${ }^{11}$ L. Balents, Europhys. Lett. 33, 291 (1996).

${ }^{12}$ M. M. Fogler, Europhys. Lett. 66, 572 (2004).
${ }^{13}$ O. Ciftja and C. Wexler, Phys. Rev. B 65, 045306 (2001).

${ }^{14}$ E. H. Rezayi and F. D. M. Haldane, Phys. Rev. Lett. 84, 4685 (2000).

${ }^{15}$ R. H. Morf, Phys. Rev. Lett. 80, 1505 (1998).

${ }^{16}$ S. M. Girvin, A. H. MacDonald, and P. M. Platzman, Phys. Rev. Lett. 54, 581 (1985); Phys. Rev. B 33, 2481 (1986).

${ }^{17}$ D. Yoshioka, The Quantum Hall Effect (Springer-Verlag, Berlin, 2002).

${ }^{18}$ N. Read and E. H. Rezayi, Phys. Rev. B 84, 085316 (2011).

${ }^{19}$ E. Fradkin and S. A. Kivelson, Phys. Rev. B 59, 8065 (1999).

${ }^{20}$ A. H. MacDonald and M. P. A. Fisher, Phys. Rev. B 61, 5724 (2000).

${ }^{21}$ D. G. Barci, E. Fradkin, S. A. Kivelson, and V. Oganesyan, Phys. Rev. B 65, 245319 (2002).

${ }^{22}$ E. H. Rezayi and F. D. M. Haldane, Phys. Rev. B 50, 17199 (1994).

${ }^{23}$ D. H. Lee and J. M. Leinaas, Phys. Rev. Lett. 92, 096401 (2004); A. Seidel, H. Fu, D.-H. Lee, J. M. Leinaas, and J. Moore, ibid. 95, 266405 (2005)

${ }^{24}$ E. J. Bergholtz and A. Karlhede, Phys. Rev. Lett. 94, 026802 (2005).

${ }^{25}$ H. Wang and V. W. Scarola, Phys. Rev. B 83, 245109 (2011).

${ }^{26}$ B. Yang, Z. Papić, E. H. Rezayi, R. N. Bhatt, and F. D. M. Haldane, Phys. Rev. B 85, 165318 (2012). 